\title{
Responsible Conduct Regarding Scientific Communication
}

\author{
Society for Neuroscience
}

\section{PREFACE}

The Society for Neuroscience believes that progress in understanding the nervous system benefits human welfare. Such progress depends on the honest pursuit of scientific research and the truthful representation of findings. Although recognizing that both error and differences among individuals in the interpretation of data are a natural part of the creative process, the Society for Neuroscience affirms that misconduct, in the form of plagiarism or fabrication or falsification of data, jeopardizes the success of the entire scientific endeavor. By entering the profession, neuroscientists assume an obligation to maintain the highest level of integrity in all scientific activities.

The Society for Neuroscience serves neuroscience and society at large in many ways, including publishing The Journal of Neuroscience and the Society for Neuroscience Abstracts, both of which present the results of scientific research. The editors of The Journal of Neuroscience have the responsibility to establish and maintain guidelines for accepting manuscripts submitted to them for publication. This document, Responsible Conduct Regarding Scientific Communication ("Guidelines"), derives from the Society's definition of the scope of the journal and from the editors' and reviewers' perception of the standards of quality for scientific work and its presentation. The Program Committee of the Society for Neuroscience has a comparable responsibility with respect to

These guidelines were approved by the Council of the Society for Neuroscience on November 7, 1998.

This document was prepared by the Ad Hoc committee on Responsibility in Publishing and its consultants on the basis of previous guidelines developed by the Society for Neuroscience and by other organizations, the committee's own deliberations, and the comments received from others. The present document draws heavily on guidelines developed by the editors of the Publications Division of the American Chemical Society, as revised in January 1994, and subsequently endorsed by the American Chemical Society's Committee on Publications (Ethical guidelines to publication of chemical research. Chem Rev 95:11A-13A, 1995). Many sections of those guidelines are reprinted here with little or no modification. The Society for Neuroscience thanks the American Chemical Society for permission to use their guidelines in this way. Some material also has been incorporated from the fifth edition of "Uniform requirements for manuscripts submitted to biomedical journals," prepared by the International Committee of Medical Journal Editors (JAMA 277:927-934, 1997).

The Ad Hoc committee was chaired by Michael J. Zigmond (University of Pittsburgh) and was composed of Eve E. Marder (Brandeis), Bruce S. McEwen (Rockefeller), Pasko Rakic (Yale), Gordon M. Shepherd (Yale), Solomon H. Snyder (Johns Hopkins), and David C. Van Essen (Washington University). Consultants to the committee included Floyd E. Bloom (Scripps Research Institute and Science), Marcel C. LaFollette (George Washington University), and Barbara Mishkin (Hogan \& Hartson). Additional input also was provided by several other individuals, including Mark S. Frankel (American Association for the Advancement of Science), Perry B. Molinoff (Bristol-Myers Squibb), Steven M. Paul (Eli Lilly), Drummond Rennie (University of California at San Francisco), and Diane Sullenberger (Proceedings of the National Academy of Sciences) as well as members of the Society for Neuroscience Council, members of the editorial board of The Journal of Neuroscience, and the Society's Publication Committee. Beth A. Fischer (University of Pittsburgh) assisted in both the development of the ideas and wording of the final document. Susan M. Giegel (University of Pittsburgh), Sherry Dixon (Society for Neuroscience), and Norman Lemke (Society for Neuroscience) provided staff assistance.

Individuals are encouraged to make copies of this document for general distribution as long as proper attribution is provided.

Copyright (C) 1998 Society for Neuroscience $\quad 0270-6474 / 98 / 190$ iii- $14 \$ 05.00 / 0$ abstracts appearing in the Society for Neuroscience Abstracts. The Society for Neuroscience also has established guidelines that pertain to other aspects of science, including the use of humans and other animals as subjects in neuroscience research, as well as a general policy on ethics. ${ }^{a}$

An essential feature of a professional society is the acceptance by its members of guidelines such as those developed by the Society for Neuroscience, codes that outline responsible behavior and specify obligations of members to each other and to the public. Such guidelines derive from a desire to maximize benefits to the profession as a whole, as well as to the general society, and to limit actions that might serve only the narrow self-interests of individuals. For example, the advancement of science requires that knowledge be shared, although doing so may sometimes entail foregoing some immediate personal advantage.

The present document is intended for persons engaged in the communication of research in neuroscience and provides a set of guidelines to reinforce and extend those previously provided by the Society for Neuroscience. The Guidelines are offered not in the sense that there is an immediate crisis in ethical behavior within the neuroscience community nor because we think that our community is particularly vulnerable to ethical problems. Instead, the Guidelines spring from a conviction that adherence to high ethical standards is so essential to the scientific enterprise that a definition of those standards should be brought to the attention of all concerned.

We believe that most aspects of the Guidelines are already understood and subscribed to by the great majority of the members of the Society for Neuroscience and by others engaged in neuroscience research. However, the Guidelines may be of help to those who are relatively new to research. In this respect, they may provide a useful text to encourage discussions of responsible conduct in science within graduate and postdoctoral training programs. Moreover, even well established scientists may appreciate these guidelines as an opportunity to review matters so significant to the practice of science.

The Guidelines are provided with particular reference to the Society for Neuroscience, its members, and its publications, which currently include The Journal of Neuroscience and the Society for Neuroscience Abstracts in both their print and electronic formats. Except where noted, the focus of the Guidelines is on peerreviewed research articles. However, we believe that the issues raised in this document are relevant to all writing, reviewing, and editing performed within neuroscience and related areas of investigation.

To facilitate the reading of these Guidelines, they have been divided into sections relating to (1) authors, (2) reviewers, and (3) editors of research manuscripts. In addition, sections deal with (4) the preparation of abstracts, (5) publication outside the scientific

${ }^{a}$ These policies are published together with the Instructions for Authors as part of each volume of The Journal of Neuroscience and in the Call for Abstracts for the annual meeting of the Society. 
literature, and (6) the enforcement of the Guidelines. Each section is divided into multiple subsections. These begin with an initial statement that summarizes the main point of the subsection and appears in italic. These sentences are followed by a brief paragraph discussing the rationale for the statement. In most instances, the paragraphs are followed in turn by one or more specific regulations or suggestions regarding proper conduct.

Invitation for comment. The Society for Neuroscience notes that we are in a period of considerable change with respect to publication, due in part to the increasing role of electronic means of transmitting information. For this reason, and because no document such as this can ever be complete, the Society invites comments from both members and nonmembers at any time. These can be addressed to Guidelines on Publishing, Society for Neuroscience, 11 Dupont Circle NW, Suite 500, Washington, DC 20036, or guidelines@sfn.org.

\section{SUMMARY OF POINTS}

\section{Authors of research manuscripts}

1.1. Authors are encouraged to have the first formal publication of their results be a peer-reviewed paper.

1.2. Manuscripts should be prepared to maximize clarity and accuracy of communication.

1.3. Authorship should be based on a substantial intellectual contribution.

1.4. "Honorary authorship" is inconsistent with the definition of authorship.

1.5. "Acknowledgments" provide an opportunity to acknowledge assistance that does not warrant authorship but does merit recognition.

1.6. Financial contributions to the work being reported should be clearly acknowledged, as should any potential conflict of interest.

1.7. Methods and materials should be described in sufficient detail to permit evaluation and replication.

1.8. Unique and propagatable research materials used in studies being reported must be made available to qualified scientists for bona fide research purposes.

1.9. Authors have an obligation to correct errors promptly.

1.10. All components of a research article should be subjected to peer review.

1.11. Plagiarism is unacceptable.

1.12. Fabrication and falsification are unacceptable.

1.13. All data should be presented to minimize the possibility of misinterpretation.

1.14. Authors should not engage in fragmented or duplicate publication.

1.15. Informal communication of results and ideas is encouraged.

1.16. Authors should not make personal attacks on other researchers.

1.17. Authors should not discuss with reviewers any aspect of a manuscript under evaluation.

1.18. Accounts of a researcher's publication record should be accurate.

\section{Reviewers of manuscripts}

2.1. Thorough scientific review is in the interest of the scientific community.
2.2. A thorough review must include consideration of the ethical dimensions of a manuscript as well as its scientific merit.

2.3. All scientists are encouraged to participate if possible when asked to review a manuscript.

2.4. Anonymity of reviewers should be preserved unless otherwise stated in the guidelines for authors and for reviewers, or unless a reviewer requests disclosure.

2.5. Reviewers should be chosen for their high qualifications and objectivity regarding a particular manuscript.

2.6. Reviews should not contain harsh language or personal attacks.

2.7. Reviews should be prompt as well as thorough.

2.8. Reviewers must not use nonpublic information contained in a manuscript to advance their own research or financial interests.

2.9. Information contained in a manuscript under review is confidential and must not be shared with others.

\section{Editors of scientific journals}

3.1. The sole responsibility for acceptance or rejection of a manuscript rests with the editor.

3.2. Editors should generally grant the request of an author who asks that an individual be excluded from the review of a particular manuscript.

3.3. Editors should establish a review process that minimizes bias.

3.4. Editors generally should not solicit specific research manuscripts.

3.5. Editors should subject all manuscripts of a given form to the same type of review.

3.6. Editors should provide to the authors a written rationale for editorial decisions regarding a manuscript submitted for publication.

3.7. Everyone involved in the editorial process must treat unpublished manuscripts as confidential documents.

3.8. A limited amount of information regarding a manuscript accepted for publication may be disclosed by an editor before publication in print.

3.9. Editors should correct errors in a manuscript if the errors are detected before publication or publish corrections if they are detected afterward.

3.10. Editors should handle cases of alleged misconduct at the lowest possible organizational level but usually must involve the institutions at which the research in question was performed.

\section{Abstracts for presentations at scientific meetings}

4.1. Abstracts for scientific meetings should be prepared with care.

4.2. In the absence of an editor, a specific individual should be designated to oversee the process of soliciting and publishing abstracts and to deal with any problems that may arise.

\section{Communications outside the scientific literature}

5.1. Research scientists are encouraged to communicate their ideas and results to the public.

5.2. Material prepared for the popular literature should be accurate and be given previous review by peers.

5.3. Communication outside the scientific literature is not a substitute for publication within the scientific literature. 


\section{Dealing with possible scientific misconduct}

6.1. Accusations should be dealt with at the lowest organizational level that can be effective.

6.2. If, after an initial inquiry, the editor believes that the accusations may have merit and cannot easily resolve the conflict, then the editor must notify the institutions at which the research was conducted.

6.3. If an editor reports alleged misconduct to the institutions at which the research was performed, the editor should ask to be informed of the outcome of any inquiry or investigation.

6.4. Allegations of scientific misconduct should be investigated promptly but with due attention to the rights of all individuals concerned.

6.5. Professional societies may initiate corrective and/or disciplinary actions based on a formal finding of serious misconduct related to its publications or its members.

\section{GUIDELINES}

\section{Authors of research manuscripts}

Authors are obliged to conduct research according to ethical precepts; to present an accurate account of the methods used, the results obtained, and the relevant scientific literature; and to provide an objective discussion of the significance of the research.

All authors submitting manuscripts or abstracts to any Society for Neuroscience publication are expected to abide by the guidelines in this document, as well as the regulations printed annually in The Journal of Neuroscience and in the Call for Abstracts. This includes submissions to The Journal of Neuroscience and the Society for Neuroscience Abstracts for the Society's annual meeting. The Society for Neuroscience expects its members to adhere to similar high standards when publishing in other journals and collections of abstracts.

\subsection{Authors are encouraged to have the first formal publication of their results be a peer-reviewed paper.}

Peer review of manuscripts is designed to provide both the author and the reader with an objective evaluation of a proposed research communication. It often results in modification of the original manuscript, ranging from clarification of language or figures to additional experiments or the reinterpretation of results. Thus, papers that have gone through peer review and have been accepted for publication have a value within the scientific community beyond that of other forms of communication. At several points within these guidelines, reference is made to the merit of non-peer-reviewed communication, including presentations at scientific meetings, material posted on the World Wide Web, and presentations to the media or to the lay public. However, none of these forms of communication replaces the publication of a peer-reviewed manuscript.

\subsection{Manuscripts should be prepared to maximize clarity and accuracy of communication.}

Research papers are the principal means by which ideas, data, and interpretations are conveyed to the scientific community. Papers that are poorly written take valuable time on the part of the reader to understand and may be subject to misinterpretation. Papers that are wordy also waste valuable resources.

1.2.1. Manuscripts should be well organized, be concise, and avoid ambiguity.

1.2.2. Authors should conform to the Instructions for Authors prepared by the editors of the journal to which their manuscript will be submitted.

1.2.3. If necessary, authors should seek the assistance of someone with experience in technical writing in the language being used for the manuscript. However, the authors of the manuscript retain responsibility for the accuracy of the final manuscript.

\subsection{Authorship should be based on a substantial intellectual contribution.}

It is properly assumed that all authors have had a significant role in the creation of a manuscript that bears their names. Therefore, the list of authors on an article serves multiple purposes; it indicates who is responsible for the work and to whom questions regarding the work should be addressed. Moreover, the credit implied by authorship is often used as a measure of scientists' productivity in evaluating them for employment, promotions, grants, and prizes.

1.3.1. The Society for Neuroscience believes that authorship must be reserved for individuals who have met each of the following conditions: (1) made a significant contribution to the conception and design or the analysis and interpretation of data; (2) participated in drafting the article or reviewing and/or revising it for intellectual content; and (3) approved the final version of the manuscript. (Deceased persons deemed appropriate as authors should be so included with a footnote reporting their death.)

1.3.2. Although researchers are strongly encouraged to share materials such as reagents, animals, and tissues (see 1.8), the provision of such materials in and of itself does not constitute sufficient grounds for inclusion as an author.

1.3.3. In multiauthored papers, the significance of the order in which authors are listed varies widely according to common practice in the field or to the policy established by the publisher and the journal and thus cannot reasonably be stipulated in these Guidelines. However, it is usual in neuroscience and allied fields for authors to be listed in descending order of their contribution to the paper, with the exception that the senior author is often listed last.

1.3.4. Once the list and order of authors has been established, the list and order of authors should not be altered without permission of all living authors. (Exceptions to this rule shall be limited to the demonstration of misconduct on the part of an author or failure to fulfill authorship obligations.)

1.3.5. The role of each author in the work reported may be indicated in a footnote.

1.3.6. Any part of an article essential to its main conclusions must be the responsibility of at least one author.

1.3.7. In the case of papers with multiple authors, a "corresponding" author must be designated as having responsibility for overseeing the publication process and ensuring the integrity of the final document. The corresponding author accepts the responsibility for (1) including as coauthors all persons appropriate and none inappropriate; (2) obtaining from all coauthors their assent to be designated as such, as well as their approval of the final version of the manuscript; (3) determining that permission has been obtained from each individual acknowledged in the manuscript (see 1.4); and (4) keeping all coauthors apprised of the current status of a manuscript submitted for publication, including furnishing all coauthors with copies of the reviewers' comments and a copy of the published version, as appropriate.

1.3.8. Coauthors have responsibility for work submitted under their names. They should remain knowledgeable insofar as pos- 
sible regarding the status of the manuscript, including the nature of any revisions.

1.3.9. If a manuscript is revised and resubmitted to the same journal, coauthors should be asked to reaffirm their assent to be listed as coauthors and to approve the revised version. In addition, if the manuscript is rejected or withdrawn from a journal and then submitted to a different journal, the coauthors should be asked again to affirm their assent to authorship even if no substantive changes have been made.

1.3.10. Coauthors have the right to withdraw their names from a manuscript at any time before acceptance of the manuscript by the editor. However, an author's name should not be removed from a manuscript without his or her permission or without approval of the editor in cases involving possible misconduct. Once a manuscript has been accepted for publication, no change in authorship should occur without permission of the editor.

\section{4. "Honorary authorship" is inconsistent with the definition of authorship.}

An honorary author is any individual listed as an author who has not made a substantive intellectual contribution to the work as defined in 1.3.1. Among those who would be considered honorary authors are those whose participation was limited solely to the acquisition of funding for the research; those who are chairs or directors of departments, divisions, or research groups and had no significant role in the planning, conduct, and review of the research; and those who merely supervised the collection of data. Honorary authorship is a misrepresentation, implying an intellectual contribution that was not made. It also distorts the publication record, making it a less reliable measure of productivity. Moreover, should honorary authors be unable to adequately discuss the work, this will reflect poorly on them and their coauthors. Finally, honorary authors risk associating themselves with work that may later be the subject of a misconduct investigation. If so, they will be expected to share in the responsibility for the work.

\section{5. "Acknowledgments" provide an opportunity to note assistance that does not warrant authorship but does merit recognition.}

Although only a limited number of people will qualify as authors of a manuscript (see 1.3.1), there are many other types of contributions that can or even should be acknowledged in other ways. Acknowledgment of ideas or of comments provided about a draft of a manuscript is an appropriate indication of assistance provided and also may facilitate such interactions in the future. However, because acknowledgments of intellectual contributions may be interpreted by readers as an endorsement of the conclusions of the paper, authors should offer such individuals the opportunity to decline the acknowledgment. Other types of acknowledgments that may be appropriate are those for the donation of a critical reagent or for technical support.

1.5.1. A footnote or the Acknowledgments section of a paper should be used to indicate intellectual, technical, or other contributions that do not merit authorship but are nonetheless noteworthy.

1.5.2. Individuals should be informed before the publication of any such acknowledgments and thereby given the opportunity to decline the offer.

\subsection{Financial contributions to the work being reported should} be clearly acknowledged, as should any potential conflict of interest.

Acknowledgment of financial support is expected by sponsors and may assist the funding agency in determining the impact of their contribution. Moreover, financial support from commercial sponsors may be a potential conflict of interest, which should be disclosed so that editors, reviewers, and readers can consider this in evaluating the objectivity of the report. Financial support includes the contribution, free of charge, of products such as drugs, biological materials, and devices.

1.6.1. All sources of financial support for the work described should be acknowledged in a footnote or in an Acknowledgments section of a manuscript.

1.6.2. Authors should disclose in a cover letter sent to the editor any associations that represent a potential conflict of interest. These include a current or pending relationship as a consultant for the company supporting the research or manufacturing products being tested, a financial or managerial interest in such a company, or intellectual property rights that might be affected by publication of the results of the research reported in a manuscript. On receipt of this information, an editor may require that a footnote disclosing the potential conflict be added to the manuscript.

1.6.3. Authors should ensure that no contractual relations or proprietary considerations exist that would restrict the dissemination of their findings. More fundamentally, researchers should seek advice from their institutions before entering into agreements that might prevent or unduly delay publication of their research results. It is generally accepted that there may be a brief delay (e.g., 30-60 days) for the sponsor to review a manuscript and prepare a patent application. However, it is not acceptable for an academic scientist to permit an outside organization to hold veto power over publication. Should any such restrictions exist, however, they should be disclosed to the editor. On receipt of this information, an editor may choose to return the manuscript.

\subsection{Methods and materials should be described in sufficient detail to permit evaluation and replication.}

In science it is essential that other researchers be able to evaluate and, if they wish, to replicate published observations. This enables researchers to build on the work of each other, thus permitting the efficient use of resources.

1.7.1. A research article should contain sufficient detail and reference to public sources of information in a format appropriate to the journal's style and policy to allow a knowledgeable scientist to evaluate and replicate the work reported.

1.7.2. The source of all materials and significant items of equipment should be clearly indicated, including those materials that are not commercially available.

1.7.3. Any known unusual hazards inherent in the chemicals, equipment, or procedures used in an investigation should be clearly identified in the manuscript reporting the work.

\subsection{Unique and propagatable materials used in studies being reported must be made available to qualified scientists for bona fide research purposes.}

In some cases, the replication and extension of published work may require materials that are not readily available. In such instances, the authors must make every effort to provide those materials to other qualified scientists. Indeed, the failure of au- 
thors to provide such materials greatly reduces the value of their work. As noted in guidelines prepared by the National Institutes of Health (1990), "this principle requires that any unique materials that are essential for repetition of the published experiments be available to other qualified scientists."

1.8.1. Once a manuscript has been published, authors should be prepared to promptly make available to qualified scientists for bona fide research purposes all materials that were used in the reported research and are not otherwise readily available. This includes propagatable research materials (such as monoclonal antibodies, transgenic mice, and DNA probes and constructs) and, where possible, nonpropagatable materials (for example, serum antibodies). Reasonable costs associated with the production and transfer of these materials should be assumed by the recipient if the authors so request.

1.8.2. Such materials should be provided without restrictions, such as the requirement that they not be used for a particular type of experiment. Likewise, the person providing the materials should not make future authorship a condition for this provision.

1.8.3. These guidelines apply equally to those in academia and in the private sector, except that when an individual in the private sector requests materials that are intended to be used for commercialization, it is appropriate that the individual requesting the materials be asked to provide a fee for licensing purposes.

1.8.4. Authors should try to arrange to provide these materials for a significant period of time after a paper has been published.

1.8.5. Authors may, if possible, arrange to distribute materials through entities such as the American Type Culture Collection (Rockville, MD), data banks (e.g., for DNA sequences), or the Jackson Laboratory (Bar Harbor, ME).

1.8.6. In general, the editors of the Journal of Neuroscience will not accept a manuscript for publication unless the authors agree in writing to the above conditions. Editors of other journals are encouraged to do the same.

1.8.7. Authors who use materials that they obtain from another source should endeavor to have those materials made available to other researchers.

1.8.8. In rare instances, considerations of time, money, or personnel may make sharing of materials impossible. In each such case the authors must explain these circumstances in a cover letter submitted with the manuscript, indicating that the authors are prepared to make every effort to assist others in creating their own materials. The editors of the journal may then determine whether to accept the manuscript for review. The editors of The Journal of Neuroscience will make such determinations on a caseby-case basis.

1.8.9. Certain considerations may lead authors, particularly those in the private sector whose work is not supported by public funds, to wish to delay providing compounds being developed as therapeutic agents. These instances must be explained and the period of delay must be defined in a cover letter submitted with the manuscript. In addition, the authors might offer to supply closely related materials (e.g., an analog to a compound). The editors can then determine whether to accept the manuscript for review.

1.8.10. If it is demonstrated to an editor that an author has failed to abide by these guidelines, the Journal of Neuroscience will refuse to publish any manuscript involving that author until the matter is corrected. Other journals are encouraged to do the same.

\subsection{Authors have an obligation to correct errors promptly.}

Once an article has been published, it remains forever within the scientific literature. Thus, care should be taken to determine that every aspect of a manuscript is correct. Occasionally, errors are not discovered until after a manuscript has been submitted or even after it has been published. Every effort should be made to correct such errors as quickly as possible. It is far preferable to do so before an article is published, because the subsequent publication of corrections-although serving a useful purpose when required-can never completely eliminate the possibility that individuals will read the original article and assume it to be accurate, having not read the correction.

1.9.1. Authors must strive to ensure that every aspect of a manuscript is correct. This responsibility does not end when a manuscript has been submitted for publication.

1.9.2. Should a significant error be discovered after the article has been submitted, is in press, or has been published, the authors must immediately contact the editor and establish how the error should best be corrected.

1.9.3. If there is a disagreement among the authors about such matters, the editor of the journal to which the manuscript was submitted must determine the proper course of action.

\subsection{All components of a research article should be subject to peer review.}

Designation as a peer-reviewed article implies that each substantive component of the published article has received editorial approval. This includes material that has been modified or added after the initial review process, as well as the deletion of material. Thus, although it may be necessary to alter a manuscript after it has been submitted (e.g., see 1.9.2), this should be done only with the consent of the editor.

1.10.1. If a manuscript has been reviewed and returned to the authors and is being sent back to the same journal in a revised form, all substantive changes in any aspect of that manuscript should be explicitly described in an accompanying note to the editor. This applies to the list and order of authors, as well as to the text, data, figures, tables, and references.

1.10.2. All substantive changes made in proofs sent to the authors after a manuscript has been accepted for publication also must be clearly identified and explained in a note to the editor.

\subsection{Plagiarism is unacceptable.}

Scientific publication is an important part of the process by which credit and priority are established for experimental work and research ideas. Duplicating without citation text previously published by others or expropriating without attribution the experimental findings, methods, or ideas of others is plagiarism and is unethical. Plagiarism undermines the system through which authors receive credit for their work and in doing so may inhibit authors from sharing their data and ideas in a timely manner, activities essential to the progress of science. In addition to denying scholarly credit, plagiarism also has potentially important legal implications for commercial development and patenting.

1.11.1. It is the responsibility of the authors to identify the source of all ideas, results, or methods quoted or offered, except those that are accepted as common knowledge.

1.11.2. In most instances, the appropriate source will be a peer-reviewed article rather than a review article, chapter, or book. When a secondary source is used to supplement a primary source, it should be identified as such (e.g., "also see review by Jones, 1992"). Abstracts, presentations at meetings or seminars, 
and material placed on a Web site also should be cited appropriately.

1.11.3. Information obtained privately, as in conversation, correspondence, or discussion with third parties, should not be used or reported in the author's work without explicit permission from the source of the information (who should then be cited as providing a personal communication). Some journals may require that written permission be obtained.

\subsection{Fabrication and falsification are unacceptable.}

It is essential that researchers and others be able to trust the validity of published data. That trust permits researchers to build on previous observations and thus facilitates the progress of science. It also allows individuals to form opinions and make policies based on those observations. Data that have been fabricated or falsified contaminate the scientific literature, greatly diminishing the value of this resource for researchers and others in the community. Moreover, such fraudulent actions undermine society's trust in the scientific enterprise.

1.12.1. No data may be communicated in an abstract, oral presentation, or publication that have not actually been collected or observed (fabrication), nor may data be altered in any way (falsification) other than by mathematical transformations that are commonly accepted or clearly explained in the manuscript. This includes numerical data, as well as visual images such as photomicrographs and gels.

1.12.2. Data that clearly deviate from all others of the same type as demonstrated by an appropriate statistical test or some other generally accepted criterion may, however, be eliminated from the data set. It may be appropriate to indicate such deletions within the manuscript.

1.12.3. All data and analyses for research reported in abstracts, articles, and oral presentations should be maintained in a retrievable form for at least 3 years after publication or presentation to permit examination and reanalysis.

\subsection{All data should be presented to minimize the possibility of misinterpretation.}

The prohibition against misrepresenting observations extends beyond fabrication and falsification. Data also must be presented in such a form that they will not be readily subject to misinterpretation.

1.13.1. Authors are obligated to present their data in a form that minimizes the chance that readers will be misled about what was actually observed.

1.13.2. This is particularly important when data transformations are used or when graphical illustrations include axes that do not begin at a standard origin (usually 0,0 ).

1.13.3. All statistical tests used to analyze data must be used knowledgeably, ensuring that the requirements of the tests are satisfied by the data set to which they are applied. Authors not well versed in the statistical procedures appropriate to their research are expected to have consulted an individual with the necessary expertise.

1.13.4. In general, complete genomic and cDNA sequences should be submitted as part of a manuscript in which such material is used. By the time a paper is sent to press, genomic and cDNA sequences should be deposited in a database generally accessible to the biomedical community; the sequence accession number should be provided in the manuscript. The editor should consider exceptions to this policy only on an individual basis.

\subsection{Authors should not engaged in fragmented or duplicate publication.}

Research reports should be neither duplicated nor unduly fragmented. Journal space is a precious resource created at considerable cost. Authors therefore have an obligation to use it wisely and economically. In addition, duplicate publication may give the misleading impression that the previously reported research has been replicated.

1.14.1. It is improper for authors to submit a manuscript describing essentially the same research simultaneously to more than one peer-reviewed research journal.

1.14.2. When submitting a manuscript for publication, authors should inform the editor of any related manuscripts under editorial consideration or in press and describe the relationships of such manuscripts to the one submitted. Copies of these manuscripts should also be supplied to the editor.

1.14.3. Authors contemplating the preparation of two related manuscripts should consider whether a single paper would be more cohesive and informative than two papers without being excessively long.

1.14.4. In general, data should never be published in more than one research article (but see 1.15). In this context, "data" refers to the full range of experimental observations, from a single value to an entire figure or table, and includes data from both control and experimental groups.

1.14.5. Authors may occasionally wish to submit a full-length research article that expands on a previously published brief preliminary account (sometimes termed a "communication" or "letter") of the same work. When the full-length research article is submitted, the editor should be apprised of the earlier publication, and the preliminary account must be cited as such in the manuscript.

1.14.6. It may sometimes be useful to include in a manuscript previously published data from a subject or group for the purpose of comparison. Also, in some cases it is helpful to have previously published data included in a new manuscript as part of a data set that is gradually developing in the course of a longitudinal study. In such cases, the duplicated data must clearly be identified as such, and citations to the previously published work must appear in the new manuscript.

\subsection{Informal communication of results and ideas is encouraged.}

Informal communication of preliminary results and ideas by meetings and abstracts before peer-reviewed publication has always been accepted and, indeed, encouraged as being in the best interest of the scientific community. It not only provides a prompt exchange of information but also often generates feedback to the authors, thereby improving the final, formal publication in peerreviewed form. The Society believes that such informal exchanges should continue to be encouraged rather than be limited by restrictive publication policies. However, new areas of communication-notably electronic dissemination of information-are now rapidly evolving, and the Society for Neuroscience will continue to monitor these areas and develop or modify its policies as appropriate.

1.15.1. Although authors are encouraged to have the first formal publication of their results be a peer-reviewed paper (see 1.1), informal communication of preliminary results and ideas is encouraged. In this context, informal communication includes presenting material at scientific meetings, posting material for a 
limited period of time on a Web site, and exchanging prepublication drafts of manuscripts.

1.15.2. When distributing information in an informal manner, whether by print or electronic means, it is advisable for authors to indicate clearly both its status with regard to publication and the date on which it was prepared and/or posted. Moreover, authors should be mindful of the fact that once a manuscript has been published, the copyright for all text, tables, and figures generally reverts to the publisher. Under such circumstances, continued distribution of any of this material by print or electronic means can only be done with permission of the publisher.

1.15.3. It is the policy of the Society for Neuroscience that such informal presentation of preliminary research results, including specific figures, does not in and of itself preclude publication as a peer-reviewed research article (but see 1.15.4).

1.15.4. The Society for Neuroscience notes that many publishers enforce policies that are different from its own with regard to material that has already been presented in an informal manner. For example, at present many journals refuse to consider material previously distributed electronically. In addition, it is possible that in the opinion of a reviewer or an editor, excessive previous communication of a result may detract from the value of republishing a particular result.

1.15.5. If a manuscript includes, in whole or in part, material previously presented in some other context, whether via print or electronic distribution or in a scientific meeting, the authors should acknowledge such presentation in a format appropriate to the journal and should obtain any permissions necessary for publication of that material. The responsibility for such citations applies both to the original author of the previously presented material and to any others who might wish to make use of that material in the preparation of a manuscript.

\subsection{Authors should not make personal attacks on other researchers.}

Within the Introduction and Discussion sections of a research article, authors relate their findings to those already in the scientific literature. This process may sometimes justify criticism, even severe criticism, of the work of another scientist. However, it is essential to the collegial nature of science that such criticism be made in a civil manner and should never involve personal attacks.

\subsection{Authors should not discuss with reviewers any aspect of a manuscript under evaluation.}

To maximize the unbiased nature of the review, the evaluation process should proceed without any interaction between authors and reviewer except through the editor.

1.17.1. Communications between authors and reviewers should be made only through the editor or a designated editorial assistant. Authors should not discuss their manuscript with a reviewer while it is under review.

1.17.2. Authors and reviewers should continue to refrain from discussing the review with each other after a review is complete.

1.17.3. Under no circumstances should an author allow an opinion rendered by a reviewer to influence the author's future actions regarding that reviewer except that an author might choose to request that a given reviewer not be asked to evaluate the author's future manuscripts.

\subsection{Accounts of a researcher's publication record should be accurate.}

The record of publication that occurs in the bibliography for a paper, on a résumé or curriculum vitae, or as part of an applica- tion for funding, often serves as an important measure of the quality and quantity of an individual's scientific accomplishments. Inaccuracies can lead to the assignment of inappropriate credit. They also can waste the time of individuals seeking the cited article.

1.18.1. When referring to one's publications or manuscripts, accurate references to the published article should be provided, or depending on the circumstances, it should be labeled as "submitted" or "in press."

1.18.2. A manuscript should not be designated as "submitted" until it has been mailed or delivered to an editor for possible publication. Moreover, "in press" (or "accepted") implies that a formal communication has been received indicating that the manuscript has been accepted and no further changes will be required. "Published" means that the article is now available in an archival form.

\section{Reviewers of manuscripts}

Peer review is an essential step in the publication process and, therefore, in research. It helps ensure that published articles describe experiments that focus on important issues and that the research is well designed and executed. In addition, it serves to promote the presentation of methods in sufficient detail to permit replication, data that are unambiguous and properly analyzed, and conclusions that are supported by the data. Finally, it promotes the proper citation of previous literature. In these ways peer review serves as a safeguard for both the authors and the readers.

\subsection{Thorough scientific review is in the interest of the scientific community.}

Although readers of the scientific literature must judge the quality of a research article for themselves, the peer review system is an extremely valuable safeguard. First, it allows readers some degree of confidence regarding the quality of the article, which is particularly important in areas with which they are not familiar. Second, it reduces the time spent reading a paper that fails to conform to generally accepted standards. Thus, it is essential that reviewers carefully evaluate a manuscript, a process that often requires several hours. A thorough review should objectively judge all aspects of the manuscript.

2.1.1. Individuals should take the time necessary to thoroughly evaluate a manuscript they have agreed to review.

2.1.2. A reviewer should consider the quality and significance of the experimental and theoretical work, the completeness of the description of methods and materials, the logical basis of the interpretation of the results, and the exposition with due regard to the maintenance of high standards of communication.

2.1.3. Reviews should include constructive suggestions for revision, including, if appropriate, indication of where statements may require additional reference to the published literature.

\subsection{A thorough review must include consideration of the ethical dimensions of a manuscript as well as its scientific merit.}

It is essential that experiments be conducted and reported in an ethical manner. Although the primary responsibility for this assurance lies with the authors, the reviewer has a critical role to play in safeguarding the integrity of the scientific literature.

2.2.1. A reviewer must consider the ethical dimensions of a manuscript and should advise the editor of any suspicions of violations of ethical standards in the research or the reporting. 
The editor should then relay appropriate questions to the authors in a timely manner.

2.2.2. The issues for consideration include but are not limited to the following: the unethical treatment of animals and human subjects, fabrication or falsification, the improper analysis of data, the use of misleading graphics, duplicate publication, and improper or omitted citation of the work of others (including plagiarism).

2.2.3. A reviewer should expect authors to meet the highest scholarly standards. It is thus appropriate for a reviewer to comment on an author's failure to cite relevant work by other scientists, bearing in mind that complaints that the reviewer's own research was insufficiently cited may seem self-serving.

\subsection{All scientists are encouraged to participate if possible when asked to review a manuscript.}

Each year, many thousands of manuscripts that are related to neuroscience are submitted to journals for consideration. Distributing the responsibility for reviewing these manuscripts as broadly as possible helps to provide expertise in a variety of areas and a diversity of opinion; it also minimizes the burdens assumed by diligent individuals.

\subsection{Anonymity of reviewers should be preserved unless otherwise stated in the guidelines for authors and for reviewers or unless a reviewer requests disclosure.}

Both authors and reviewers should observe the policies for confidentiality as set by the journal concerned, noting that such policies can differ significantly among journals. Most journals in neuroscience and related fields do not identify reviewers to the authors of manuscripts, because it is felt that disclosure might inhibit adequate review. However, those journals usually reveal the identity of the authors to the reviewer, because it is assumed that this information assists in evaluating a manuscript's quality. For example, it may be important to know whether a given author has experience with a particular technique. When such imbalance in information exists, it should not be permitted to affect either the quality or the confidentiality of the review process.

2.4.1. Reviewers should not communicate with authors about a manuscript under consideration. Likewise, authors should not initiate such a communication with a reviewer (see 1.17) but instead should communicate only with the editor. If an author persists in attempting to communicate with a reviewer, that reviewer should notify the editor.

2.4.2. The Journal of Neuroscience will not disclose the identity of reviewers for any given manuscript except when explicitly requested to do so by the reviewer or required to do so under court order. It may, however, publish annually a list of all individuals who have served as reviewers, including any individuals whose advice a primary reviewer had solicited (see 2.9.6).

\subsection{Reviewers should be chosen for their high qualifications and objectivity regarding a particular manuscript.}

Individuals who are active in the area of research addressed in a manuscript may often be the most qualified reviewers. However, for the peer review process to work effectively, authors and editors also must be assured that reviewers are impartial. For these reasons, reviewers should be sensitive to any conflict of interest or appearance of such conflict with regard to a particular manuscript that they are asked to review.

2.5.1. An individual who is asked to review a manuscript and who feels inadequately qualified to judge that manuscript should return it promptly to the editor.
2.5.2. Individuals must inform the editor of any potential conflict of interest regarding a manuscript and should decline to review the manuscript if they believe that the conflict of interest might impair their objectivity. Examples of a conflict of interest might include but are not limited to (1) a manuscript that is so closely related to the potential reviewer's work in progress that it would be difficult to ensure that the reviewer would not be influenced by reading the manuscript; (2) a manuscript that strongly supports or refutes the potential reviewer's opinions; (3) an author who has recently been associated with the potential reviewer as a mentor, student, collaborator, or protagonist; and (4) a manuscript that discusses an issue or an organization in which the potential reviewer has a financial interest.

2.5.3. If in doubt as to whether circumstances present a conflict of interest that would impair a reviewer's objectivity, the reviewer should choose one of the following options: the reviewer may (1) return the manuscript promptly without review and advise the editor of the circumstances, (2) contact the editor and defer to the editor's judgment with regard to the appropriateness of serving as a reviewer, or (3) explain to the editors the possible conflict of interest in a confidential comment that accompanies the review.

\subsection{Reviews should not contain harsh language or personal attacks.}

Reviewers need not refrain from rendering a critical judgment; indeed, this is in the best interest of science. However, reviewers should comment tactfully. Harsh language and personal attacks on the authors are unacceptable; they also may call into question the validity of the reviewer's comments.

\subsection{Reviews should be prompt as well as thorough.}

Objectivity and thoroughness are essential qualities of a review; so is promptness. Authors profit from timely feedback, as when an additional experiment or modification of a method is recommended. Moreover, priority — publishing a finding before others do so-is often an important criterion in the evaluation of an author's productivity.

2.7.1. Reviewers must be allowed and should take the time necessary to provide a thorough review. They also should submit their evaluation of the manuscript in a timely manner. The Society for Neuroscience considers that 2 weeks is usually an adequate period of time to complete the review of a full-length manuscript.

2.7.2. Should a reviewer receive a manuscript at a time when circumstances preclude prompt attention to it, the unreviewed manuscript may be returned immediately to the editor. Alternatively, the reviewer may notify the editor of probable delays, propose a revised deadline for the review, and defer to the editor's judgment regarding the acceptability of a delay.

\subsection{Reviewers must not use nonpublic information contained in a manuscript to advance their own research or financial interests.}

The resources necessary for research are scarce and are awarded in large part to those individuals who are credited with the best ideas and the highest productivity. Yet, authors willingly submit manuscripts for review before receiving credit for their work. Thus, it is essential that reviewers not abuse their privileged positions by attempting to benefit from their advanced access to new ideas, methods, or data.

2.8.1. Reviewers should not use any information, arguments, or interpretations contained in a manuscript under consideration to 
advance their research unless the information has been made publicly available through another source, such as an abstract or a presentation at a meeting, a stock offering, or a new article.

2.8.2. There is one exception to this rule: If information obtained during the review of a manuscript indicates that some of the reviewer's own research is unlikely to be successful, it would be ethical for the reviewer to discontinue the research.

2.8.3. Individuals should not buy or sell stock in a company whose product figures prominently in a manuscript they are reviewing until after the manuscript is published or the information contained in the manuscript becomes publicly available through some other means. Neither should they buy or sell stock in a competitor based on nonpublic information in a manuscript they have reviewed.

\subsection{Information contained in a manuscript under review is confidential and must not be shared with others.}

The rationale prohibiting reviewers from profiting from their advanced access to a manuscript also dictates that reviewers treat the document as confidential. If it is in the best interests of the review process to obtain additional advice, this must be done with careful attention to matters of conflict of interest and confidentiality and in conformity with the journal's policies.

2.9.1. Reviewers, as well as their administrative staff who deal with the manuscript, should neither share nor discuss a manuscript with others, except in special cases when additional specific advice is necessary to provide a thorough review, and then only if consistent with instructions from the editor.

2.9.2. In the event that outside advice is deemed necessary, the reviewer should request permission from the editor if journal instructions so indicate. This will allow the editor to determine whether the authors of the manuscript have requested that the individual in question not be assigned as a reviewer.

2.9.3. If the designated reviewer does consult additional colleagues, the number of such individuals should be kept to a minimum. Moreover, it is the reviewer's responsibility to ensure that each such individuals are aware of all relevant aspects of these Guidelines and other pertinent policies for the journal concerned, especially those dealing with conflict of interest and confidentiality.

2.9.4. The practice of distributing a manuscript under review to a number of trainees purely for instructional purposes is a breach of confidentiality and is therefore inappropriate.

2.9.5. Unless otherwise agreed to by the editor or indicated in the instructions, the person to whom the manuscript was originally sent bears ultimate responsibility for the accuracy of the review and for ensuring that additional readers do not compromise the integrity of the review process.

2.9.6. When outside advice is sought, the initial reviewer should indicate the identity of all consultants when submitting the review.

2.9.7. Reviewers should be mindful of the fact that unpublished manuscripts remain the property of the authors until a copyright agreement between the authors and the publisher has been signed.

\section{Editors of scientific journals}

The review process needs a director, such as an editor (or editors) charged with ensuring the high quality of all manuscripts accepted for publication and with maintaining the objectivity and confidentiality of the process used to make that determination.

\subsection{The sole responsibility for acceptance or rejection of a manuscript rests with the editor.}

The primary task of the editors of any journal is to ensure that all manuscripts are evaluated primarily with regard to the importance and quality of the work reported and its relevance to the journal's mission. The editors of The Journal of Neuroscience include the Editor-in-Chief (who has ultimate responsibility for the Journal), the Senior Editors (who make final decisions on manuscripts), and the Reviewing Editors.

3.1.1. An editor may reject a manuscript without additional opinions if it is deemed to be (1) inappropriate as to subject matter or format, (2) of poor quality, or (3) of inadequate significance. This decision, based primarily on the manuscript as submitted, also may take into account the editor's assessment of the possible impact of revisions by the author.

3.1.2. In the case of a conflict of interest (see 1.6), an editor may request that the authors include a statement to this effect in the manuscript before it can be reviewed or accepted for publication.

3.1.3. For manuscripts that pass this initial screening, responsible and prudent exercise of editorial responsibilities normally requires that the editor seek advice from reviewers as to the appropriateness of the manuscript for publication in the journal for which it is being considered.

3.1.4. Editors should endeavor to select reviewers who possess appropriate expertise and exercise sound judgment. Editors then should ensure that the reviewers understand their responsibilities, including those regarding confidentiality and the timely preparation of an unbiased review.

3.1.5. Editors are under no obligation to reconsider a manuscript they have rejected. However, an editor may offer the authors an opportunity to respond to criticisms and/or to prepare a revised version. In this case, the editor should permit the authors a reasonable but limited period of time in which to do so.

3.1.6. Editors should hold authors to a high standard with regard to the citation of appropriate literature, emphasizing the use of initial, peer-reviewed references whenever possible. However, editors should not encourage authors to cite the editors' journal merely to enhance that journal's reputation.

\subsection{Editors should generally grant the request of an author who asks that an individual be excluded from the review of a particular manuscript.}

There are legitimate reasons for authors to request that particular individuals not review their manuscripts. For example, the individual may be a competitor in a rapidly moving field or may have previously demonstrated an inappropriate bias against the author.

3.2.1. Authors may request that the editor not involve certain individuals in the review of their manuscript. When possible the editor should grant this request. However, the editor may decide to use one or more of these reviewers if the editor believes that their expertise is critical to the fair consideration of the manuscript. If an editor does use a reviewer despite an author's objection, the editor should seek the opinions of additional reviewers.

3.2.2. Authors may indicate in their cover letter that the manuscript should be returned to them rather than be reviewed by a particular individual. An editor should respect this request.

\subsection{Editors should establish a review process that minimizes bias.}

Science flourishes best when publication in peer-reviewed journals is based solely on the quality and scientific importance of manuscripts and their relevance to the mission of those journals. 
This applies to all journals, regardless of whether they are published by a nonprofit scientific organization, academic institution, or commercial firm.

3.3.1. An editor should give unbiased consideration to all manuscripts offered for publication, judging each on its merits without regard to any personal characteristic of the authors. Such irrelevant characteristics include age, ethnicity, gender, institutional affiliation, nationality, race, religion, seniority, and sexual orientation.

3.3.2. Editors should urge reviewers to be objective in their evaluation of a manuscript.

3.3.3. Except in the case of signed editorials, editorial responsibility for any manuscript with which the editor has a potential conflict of interest should be delegated to another qualified person, such as another member of the editorial board or senior editorial staff of that journal. This may be necessary, for example, when a manuscript under review is authored by the editor or someone at the editor's institution or a present student or collaborator, is closely related to the current or past research of an editor, or may be related to an editor's financial interests. (See related comments for reviewers in 2.5.1.)

3.3.4. In some cases, it may be appropriate to inform the authors about the editor's current or planned research in a given area to avoid any possible misunderstandings concerning the origin of the editor's ideas.

3.3.5. Editors should ensure that throughout the review process the intellectual independence of authors is respected and room is left for well reasoned differences in opinion.

\subsection{Editors generally should not solicit specific research manuscripts.}

Editors are encouraged to maintain or improve the quality of their journal by carefully reviewing submitted manuscripts and by other means such as providing a high-quality format. They may also wish to make frequent announcements of the journal's mission. However, when editors solicit a particular manuscript for their journal, they jeopardize their ability to provide - and to be seen as providing-the objective evaluation that is the core of their responsibility.

3.4.1. Editors may wish to solicit opinion pieces or editorials. However, they should not request submission of a particular research manuscript by a particular author, lest it be implied that the article will receive favored treatment during review. They also should never suggest that a manuscript will be accepted until the review process has been completed (see 3.5.4).

3.4.2. Editors may, however, advertise their general interest in a type of manuscript or otherwise publicize their journals.

3.4.3. If a different editorial policy applies to any manuscripts within a journal, this should be stated explicitly in the guidelines to the authors or some other suitable place within the journal. Unless this different policy affects a large percentage of the published articles, editors also are encouraged to indicate the policy in a footnote to the specific article to which it applies.

\subsection{Editors should subject all manuscripts of a given form to the same type of review.}

If readers are to assume that publication indicates a manuscript has achieved the standards set by a given journal, then all articles within that journal (or a particular section of the journal) must receive an equivalent review. Moreover, because special credit is provided to the individual who publishes a finding first, editors should endeavor to have all manuscripts reviewed and published with the same degree of promptness.

3.5.1. Editors should consider manuscripts submitted for publication with all reasonable speed. Likewise, editors should strive to publish manuscripts in chronological order of acceptance.

3.5.2. When publication may be delayed by some production detail-such as the failure of authors to return page proofs promptly or problems with the reproduction of a figure-the authors should be informed of this delay.

3.5.3. In instances in which these guidelines are not adhered to, as in the case of a manuscript that has been placed on a "fast track," the editors should state explicitly in a footnote how the manuscript was handled. Alternatively, the editors may wish to place such papers in a section of the journal explicitly designated for this purpose.

3.5.4. Authors should never be given any assurance of a positive outcome of the review process until that process has been completed. This requires complete and thorough evaluation of the submitted manuscript (see Section 2) and usually involves input from two or more reviewers other than the editor (see 3.1.3)

\subsection{Editors should provide to the authors a written rationale for editorial decisions regarding a manuscript submitted for publication.}

It is essential that the scientific community, including each individual author, has as much confidence in the editorial process as possible. Thus, a written explanation of an editorial decisionusually including the comments of reviewers-is essential. Moreover, such feedback can play an important role in encouraging good science and manuscripts of high quality in the future.

3.6.1. Editors should provide the corresponding author with a copy of the reviewers' comments regarding a manuscript.

3.6.2. Before forwarding a reviewer's comments to an author, the editor may delete any inappropriately harsh language or personal attacks included in the review. The need for these deletions should be brought to the attention of the reviewer. Such language or attacks should not influence the editor's decision regarding the manuscript, although it may require the editor to seek input from an additional reviewer.

\subsection{Everyone involved in the editorial process must treat unpublished manuscripts as confidential documents.}

Until a manuscript is published, editors and members of their editorial staffs are expected to treat it as a privileged document (see 2.8 and 2.9).

3.7.1. Unpublished research ideas, information, arguments, or interpretations disclosed in a submitted manuscript should not be used in an editor's own research or for the personal financial gain of an editor or anyone associated with a journal. However, if information obtained during the review of a manuscript indicates that some of the editor's own research is unlikely to be successful, it would be ethical for the editor to discontinue the research.

3.7.2. The editor, the editor's staff, and the journal's staff should not disclose information about a manuscript under consideration to anyone other than those from whom professional advice is sought or as part of the normal editorial process.

3.7.3. However, an editor who solicits or otherwise arranges beforehand for the submission of manuscripts may need to disclose to prospective authors the fact that a relevant manuscript by another author has been received or is in preparation. This may occur, for example, during development or production of a special issue. 
3.8. A limited amount of information regarding a manuscript accepted for publication may be disclosed by an editor to the public before publication in print.

Once a manuscript has been accepted for publication, several months may elapse before it is available in print or by electronic means. However, in certain cases, it may be of value to hasten the dissemination of some or all of the contents of the article. This might occur, for example, if the article contains information important to public health.

3.8.1. After a manuscript has been accepted for publication, it is reasonable for the editor and members of the editor's staff to release information about or from the manuscript even before the manuscript appears in print.

3.8.2. With the exception of the title and authors' names, the contents of a manuscript should not normally be disclosed before publication in print or electronic form without the authors' permission unless such disclosure is part of the published policy of the journal.

3.8.3. If disclosure before publication is allowed by the journal and if the authors give permission, then an editor may release some or all of a manuscript (including tables and figures) via press release or advanced print or electronic copy.

3.8.4. In any such cases, it is important that information disclosed before publication must be made generally available. Selective and limited disclosure (e.g., to colleagues, friends, or family) is not acceptable.

\subsection{Editors should correct errors in a manuscript if they are detected before publication or publish corrections if they are detected afterward.}

Honest errors can escape detection until after a manuscript has been submitted or even published, as when a reagent subsequently proves to be less specific than originally believed or a measuring device is later shown to have been inaccurate. Occasionally, calculations are incorrect or a critical paper is discovered late. An author, a reviewer, an editor, or any other individual may raise the possibility of error. In each case, it is imperative that the editor carefully investigates the possible error once it has been pointed out. When errors significantly alter some aspect of an article, the editor and publisher should provide a means by which a correction or retraction can be made.

3.9.1. If someone other than an author brings an error or apparent error to an editor's attention, the editor should notify all authors as soon as possible and request correction.

3.9.2. If an error may significantly affect a manuscript or published article, then corrective action should be taken. If a manuscript has not yet been published, the errors should be corrected before publication, or else publication should be delayed. If the article has been published, then a report about the error should be published in the journal in which the original article appeared.

3.9.3. In the case of errors in reports that have already been published, the authors should always be given the opportunity to respond to and report the error. If the authors do not do so in a timely manner, then the editor of the journal should publish a notice of correction written by an individual of the editor's choosing.

3.9.4. All notices of correction or retraction must be published prominently in the journal in which the original report appeared and contain the full bibliographic reference to the original article or abstract. It should also be listed in the contents page and be prominently labeled (e.g., erratum, retraction, or apologia).
3.10. Editors should handle cases of alleged misconduct at the lowest possible organizational level but usually must involve the institutions at which the research in question was performed.

In rare instances, inaccuracies may have been deliberately included in a manuscript submitted for publication. Such inaccuracies could include misrepresenting data or failing to cite the source of a central concept and could constitute scientific misconduct (see the introductory remarks in Section 6). Editors may conduct an initial inquiry into apparent or alleged misconduct involving articles under consideration, in press, or published in their journals. However, editors generally do not have either the resources or the power required for significant investigatory activity. If the editor cannot easily resolve cases of alleged misconduct, the editor should refer those cases to the institutions at which the research was performed, requesting that they be informed of the outcome of any investigation (see 6.3.).

\section{Abstracts for presentations at scientific meetings}

The presentation of research findings at scientific meetings can be an extremely valuable means for the rapid dissemination of information as well as the garnering of feedback before publication. Such presentations may be preceded by the publication of an abstract.

\subsection{Abstracts for scientific meetings should be prepared with care.}

Collections of abstracts for presentations at professional meetings are themselves scientific communications. These abstracts may be used by individuals in planning their research and may even be cited in peer-reviewed papers. They are often disseminated widely and retained within scientific libraries. Because abstracts often must be submitted many months before the meeting and are usually not subjected to editorial review, the authors bear full responsibility to ensure that these communications are prepared responsibly.

4.1.1. Within the constraints of the space permitted, authors should observe the same standards for abstracts as are expected for journal articles, as stated in Section 1, above. These conditions include those that relate to authorship and acknowledgments, as well as the prohibitions against plagiarism, falsification, and fabrication.

4.1.2. Descriptions of experimental results in abstracts for meetings should be supported by existing data.

4.1.3. Authors should not make statements in abstracts about data that have not yet been collected.

4.1.4. In the absence of a formal editorial review, authors are encouraged to have their abstracts reviewed by colleagues before submission.

\subsection{In the absence of an editor, a specific individual should be designated to oversee the process of soliciting and publishing abstracts and to deal with any problems that may arise.}

Although abstracts often are not edited, it nonetheless is necessary to establish guidelines for their preparation and dissemination. In addition, standards must be established and monitored regarding responsible conduct in the preparation and delivery of these communications.

4.2.1. The organizers of any scientific meeting should designate in advance an individual responsible for overseeing the preparation of instructions for the submission of abstracts, as well as guidelines for their dissemination. In addition, an individual 
should be designated to be responsible for responding to any reports of error or allegation of misconduct.

4.2.2. In the case of abstracts submitted to the Society for Neuroscience, the responsible individual is the chair of the Program Committee, and the guidelines to be followed are those established for the editor of a journal (see Section 3). Thus, where appropriate, the term "editor" in these Guidelines should be replaced with the term "chair of the Program Committee."

\section{Communications outside the scientific literature}

Communication with the lay public through publication of research results and discussions is encouraged. Such communications help disseminate knowledge to the general community and can promote an appreciation of research in neuroscience, much of which is supported with public funds. However, these communications must be made responsibly, staying within the boundaries set by the level of understanding of the audience and the need for accuracy and responsibility. In most instances, research findings should be published or accepted for publication in a peerreviewed journal before being announced to the public.

\subsection{Research scientists should seek to communicate their ideas and results to the general public.}

Researchers are encouraged to discuss their ideas and their results with the public. This might occur through oral presentations, press releases, or articles written for the lay community or assistance and advice to those producing public communication in science. The Society for Neuroscience maintains a staff to assist its members in this regard. Researchers are cautioned, however, that federal law prohibits promoting a drug or device for indications not approved by the Food and Drug Administration.

\subsection{Material prepared for the popular literature should be accurate and be given previous review by peers.}

Scientific terminology provides the precision essential to the conduct of science yet may be unintelligible or unnecessarily complex for communicating with the general public. Scientists are encouraged to use language appropriate to their audience, even though this may result in some loss of precision. The scientist should, however, strive to keep public writing, remarks, and interviews as accurate as possible, given the constraints of effective communication, the particular medium, and the extent to which the scientist is able to control the final product or communication.

5.2.1. When communicating outside the scientific literature, researchers should adhere to the same general ethical principles that apply to research articles. This includes giving appropriate credit to others; the prohibitions against fabrication, falsification, and plagiarism; the principles that define authorship; and the requirement that potential conflicts of interest be disclosed.

5.2.2. A scientist should not publicly announce a discovery unless the experimental, statistical, and theoretical support for it is of sufficient strength to warrant publication in the scientific literature (see 5.3).

\subsection{Communication outside the scientific literature is not a substitute for publication within the scientific literature.}

Although communication of ideas and results to the lay public is strongly encouraged, this does not substitute for publication of those ideas and results in a peer-reviewed journal. Moreover, public trust in the scientific endeavor can be greatly harmed through the premature release of findings that are called into question or disproved shortly thereafter. Thus, it generally is best if the initial public announcement of a scientific finding occurs after acceptance by a peer-reviewed journal.

5.3.1. In most instances it is in the best interest of science that a finding be reviewed and accepted for publication in a peerreviewed journal before it is announced to the public.

5.3.2. Under certain circumstances an author may conclude that the public good is best served by more rapid dissemination of research findings. In such circumstances, special care must be taken to ensure that the conclusions presented to the public are well supported. If the work has not yet been subjected to formal editorial or peer review, the proposed communication should be reviewed by knowledgeable colleagues.

5.3.3. When publication of a result in the popular press precedes publication in a peer-reviewed journal, an account of the experimental work and results should be submitted as quickly as possible for publication in such a journal.

5.3.4. Researchers are cautioned that extensive disclosure of research results in the public press may preclude publication in some peer-reviewed journals. This, however, is not the view of the Society for Neuroscience (see 1.15).

\section{Dealing with possible scientific misconduct}

The importance of responsible conduct in scientific research and communication has been emphasized throughout these Guidelines. In the great majority of circumstances, those involved in such communications understand the importance of such conduct and behave appropriately. However, exceptions can occur, and when an allegation of misconduct is made, it is essential that the scientific community respond quickly and effectively. It is true that investigations of misconduct often consume valuable resources and can be very unpleasant, and that enforcement of prohibitions against misconduct can be even more costly and unpleasant. Yet, as a professional society we have a responsibility for maintaining the scientific integrity of our members and those others who communicate their research through our publications and at our annual meetings. Moreover, only by accepting this responsibility ourselves can we minimize the likelihood that individuals outside of the research enterprise will take the responsibility from us.

The Society for Neuroscience believes that each of the guidelines established herein is relevant to the responsible conduct of science. The Society also recognizes that the guidelines deal with matters of varying seriousness and that honest errors can sometimes occur. Thus, for the purposes of this document, the term "misconduct" is limited to instances of intentional fabrication, falsification, or plagiarism by authors, reviewers, or editors. Note, however, that these terms may be applied to any section of any type of communication of scientific observations.

\subsection{Accusations of misconduct should be dealt with at the lowest organizational level that can be effective.}

Editors may conduct a preliminary inquiry into alleged misconduct related to a manuscript that is in review or in press or has been published by their journal. Resolving matters at this level saves time and money and helps protect the reputations of all involved, in particular, those of individuals who are unjustly accused. However, the resolution should involve consultation with the chair of the Publications Committee and, as appropriate, the Executive Committee. 
6.2. If, after an initial inquiry, the editor believes that an accusation of misconduct may have merit, then the editor must notify the institutions at which the research was conducted.

In the United States and many other countries, responsibility for dealing with alleged misconduct lies with the institutions at which the research was performed and with the cognizant federal agencies. The institutions at which work was performed usually will have primary responsibility for considering and resolving allegations of scientific misconduct regarding that work.

There are several reasons for the primacy of institutions in the consideration of allegations of misconduct. First, most cases of alleged misconduct involve the use or misuse of funds awarded to a particular institution and/or activities performed as part of the obligations of an individual to that institution. Second, research institutions have the resources to conduct misconduct investigations and the right of access to research materials and personnel. Third, alleged misconduct in science may involve violations of ethical principles and institutional standards of conduct but not necessarily scientific misconduct as defined by law. Fourth, institutions have an interest in the conduct of their faculty, staff, and students. Thus, most institutions have policies that define scientific misconduct, procedures for investigating allegations of misconduct, and penalties for scientific misconduct as well as for false allegations of misconduct.

6.2.1. The Society for Neuroscience supports the principle that institutions engaged in research should have effective procedures for dealing with allegations of scientific misconduct.

6.2.2. Evidence of possible misconduct regarding a manuscript should be reported to the research integrity officers of the institutions at which the research was conducted.

6.2.3. All possible care should be taken to handle these matters in a manner that protects the rights and reputations of everyone concerned (see 6.4).

\subsection{When an editor reports alleged misconduct to the} institutions at which the research was performed, the editor also should ask to be informed of the progress and outcome of any inquiry or investigation.

Those responsible for the publication process also have an interest in maintaining the highest standards of conduct. Despite the central role of the research institution in dealing with misconduct, those responsible for the publication of a research article or abstract also should participate, if requested, in such inquiries. The Society of Neuroscience has special responsibility for matters related to The Journal of Neuroscience, the Society for Neuroscience Abstracts, and any of its other publications in either print or electronic form. Moreover, the Society also has a special interest in the professional conduct of its members, including conduct that does not directly concern Society publications.

6.3.1. If a preliminary inquiry justifies a formal investigation by an institution or federal agency, the editor of the journal to which the manuscript was submitted (or the individual responsible for the meeting abstract) should ask to be notified of that investigation early in the process. The editor also should request to be kept informed of its progress and told about its conclusions.

\subsection{Allegations of scientific misconduct should be investigated promptly but with due attention to the rights of all individuals concerned.}

Misconduct in research threatens the scientific enterprise and should be investigated promptly and thoroughly. However, it is essential that such investigations respect the rights of both the accused and the individual making the accusation.
6.4.1. Individuals who allege misconduct should be asked to provide their evidence to the editor and/or authors' institutions.

6.4.2. Authors are expected to cooperate fully with misconduct investigations, including providing access to original data and laboratory notebooks.

6.4.3. Individuals who allege misconduct are encouraged to allow their identities to be made known to the investigating institution. However, should they choose not to do so, this request should be respected.

6.4.4. At all stages, every effort should be made to ensure that the process is fair and just, both for the accused and the individuals making the allegation.

6.4.5. The accused should be considered innocent of wrongdoing until the evidence indicates otherwise. However, an editor may delay publication of a challenged paper pending the outcome of an investigation.

6.4.6. Accusers who bring forward allegations in good faith should not be subjected to retaliation, even if no misconduct is found.

6.4.7. The entity investigating the accusation should complete that investigation as quickly as possible, consistent with the need for a thorough and impartial inquiry.

6.4.8. Knowledge that an investigation is being or has been conducted, as well as any information collected in the process, should be restricted to the absolute minimum number of persons necessary and treated with strict confidentiality, even after the investigation is complete. However, information regarding the investigation and its findings should be released in cases in which misconduct has been determined to have occurred or when knowledge that an investigation is being conducted has become generally known and an accused scientist is exonerated.

\subsection{Professional societies may initiate corrective and/or} disciplinary actions based on a finding of serious misconduct related to its publications or its members.

In some cases it may be appropriate that a professional society take further actions regarding a finding of scientific misconduct by an editor, research institution, granting agency, or court of law. The specific guidelines that follow refer to the Society for Neuroscience. Other professional societies may wish to develop procedures in accordance with their own structure.

6.5.1. Once an investigation of misconduct is concluded by the relevant institution or agency, the individual responsible for the publication (e.g., the editor of a journal) should be informed of the outcome. That individual should then inform the Executive Committee of the Council of the Society for Neuroscience of the outcome, including any administrative or disciplinary action that has been taken by the institution or agency.

6.5.2. In the case of investigations related to manuscripts or abstracts submitted to The Journal of Neuroscience or the Society for Neuroscience Abstracts, recommendations for sanctions should be made by the Executive Committee of the Society for Neuroscience to the Editor-in-Chief of The Journal of Neuroscience or the Chair of the Program Committee of the Society for Neuroscience, respectively, and to its Publications Committee. These individuals will then determine the appropriate action in association with the Executive Committee.

6.5.3. Some investigations will involve publications submitted to a non-Society publication by a member of the Society. Findings of misconduct should be reported to the Executive Committee of the Society by anyone who becomes aware of them. The Executive Committee then will determine the appropriate action. 
6.5.4. If an investigation concerning a published article or abstract determines that the article contains a serious error, then a correction or retraction must be published prominently in the journal or abstract collection in which the original report appeared and contain the full bibliographic reference to the original article or abstract. It should also be listed in the contents page and be prominently labeled (e.g., erratum, retraction, or apologia) (also see 3.9.4).

6.5.5. If the article or abstract was authored by more than one individual and some of those individuals are found to be innocent of misconduct, this should be made clear in the published statement.

6.5.6. Any coauthors not found to be guilty of misconduct should be invited to participate in the preparation of the correc- tion or retraction and/or to add an indication of their agreement to the statement. However, such authors should not be permitted to block publication of the statement.

6.5.7. If it is determined that allegations were not made in good faith or were maliciously motivated, disciplinary action may be recommended for those responsible by the Executive Committee in association with the Editor-in-Chief of the Journal of Neuroscience or the chair of the Program Committee. This recommendation is made to the Council of the Society for Neuroscience, which then determines the course of action to be taken.

6.5.8. The Council of the Society for Neuroscience retains the right to consider additional action. In accordance with the bylaws of the Society, this action may include expulsion from the Society. 